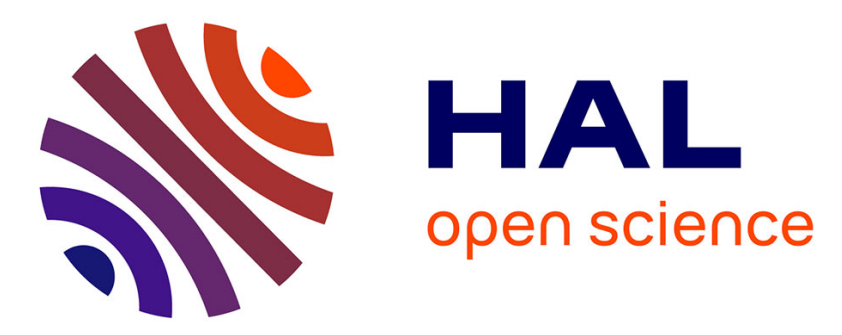

\title{
Flight-path tracking control of a transportation aircraft : Comparison of two nonlinear design approaches
}

\author{
Lili Duan, Wen Chi Lu, Felix Mora-Camino, Thierry Miquel
}

\section{To cite this version:}

Lili Duan, Wen Chi Lu, Felix Mora-Camino, Thierry Miquel. Flight-path tracking control of a transportation aircraft: Comparison of two nonlinear design approaches. DASC 2006, 25th IEEE/AIAA Digital Avionics Systerms Conference, Oct 2006, Portsmouth, United States. pp 1-9, 10.1109/DASC.2006.313702 . hal-00938130

\section{HAL Id: hal-00938130 \\ https://hal-enac.archives-ouvertes.fr/hal-00938130}

Submitted on 27 May 2014

HAL is a multi-disciplinary open access archive for the deposit and dissemination of scientific research documents, whether they are published or not. The documents may come from teaching and research institutions in France or abroad, or from public or private research centers.
L'archive ouverte pluridisciplinaire HAL, est destinée au dépôt et à la diffusion de documents scientifiques de niveau recherche, publiés ou non, émanant des établissements d'enseignement et de recherche français ou étrangers, des laboratoires publics ou privés. 


\title{
FLIGHT-PATH TRACKING CONTROL OF A TRANSPORTATION AIRCRAFT: COMPARISON OF TWO NONLINEAR DESIGN APPROACHES
}

\author{
L. Duan ${ }^{1}$, ENAC/UT2, Toulouse, France \\ W. Lu ${ }^{2}$, F. Mora-Camino ${ }^{3}, E N A C / D G A C$, Toulouse, France \\ T. Miquel ${ }^{4}$, DSNA/DTI/DGAC, Toulouse, France
}

\begin{abstract}
For transport aircraft, the primary control objective for an autopilot system engaged during approach and landing is relative to the flight-path tracking on the basis of highly simplified linear models of flight dynamics. The dynamics governing the flight-path of an aircraft are in general highly nonlinear and involve complex physics for which no accurate models are available. In the past decades, some new nonlinear control design techniques appeared of interest for the development of improved control schemes for the flight-path tracking mode. In this communication, two nonlinear control techniques are used for the design of flight-path tracking control laws for a transport aircraft. A first control technique adopts a classical nonlinear dynamic inversion control scheme where enhanced robustness is introduced, and a second control scheme based on the backstepping technique is developed. These two approaches are compared in terms of applicability, complexity and robustness with respect to modeling inaccuracies and external perturbations. Finally, simulation results are displayed and discussed.
\end{abstract}

\section{Nomenclature}

$V_{T}$ : true aircraft speed,

$\alpha$ : angle-of-attack, $\gamma:$ vertical path angle, $\theta:$ pitch angle,

$q$ pitch angular rate,

$\delta_{e}$, : elevator setting,

$L, T$ : lift and thrust forces,

\footnotetext{
${ }^{1}$ Ph.D student

${ }^{2}$ Postdoctor

${ }^{3}$ Professor

${ }^{4}$ Researcher
}

$M$ : pitch moment, $I_{y}$ : y-axis inertial moment, $\rho$ : air density, $\bar{c}$ : mean wing chord, $S$ : reference area,

$C_{L_{0}}, C_{L_{\alpha}}, C_{L_{\delta}}, C_{m_{0}}, C_{m_{\alpha}}, C_{m_{\delta}}, C_{m_{q}}, C_{m_{\dot{\alpha}}}$ : non

dimensional aerodynamic parameters,

$m$ : aircraft mass, $g$ : gravitational acceleration

\section{Introduction}

The application of nonlinear control techniques to flight control problems has persistently drawn considerable attention in the past several decades [1] [2]. More recently, systematical analysis tools have been developed to enhance their applicability. Nonlinear dynamics inversion control is already a popular nonlinear technique used to tackle flight control problems. In the last two decades, the nonlinear dynamics inversion control approach has been well established in theory and has drawn considerable attention for its applications to flight control problems. This technique avoids the tedious gain scheduling problem and the constrained stability for small maneuvers as well. Due to the principle of dynamics inversion involving canceling the nonlinearities, it strongly relies on rather precise nonlinear models. This may produce limited performance when applied to systems with only approximate empirical nonlinear models. Some research works have introduced the use of neural networks to build up accurate numerical models over a region of interest. Contrarily to the nonlinearities cancelling approach of nonlinear dynamics inversion technique, the backstepping technique intends to take advantage of the 
nonlinearities and this mainly to guarantee the global stability of the controlled system dynamics. It appears specially suited to quite common cascaded forms. Its extensions to adaptive control and its integration with other nonlinear control techniques seem to enhance its robustness and to broaden its field of application.

The main aim of this paper is to assess the respective performances of the nonlinear dynamic inversion technique and of the backstepping technique applied to the flight-path tracking control problem.

First, control law synthesis based on the nonlinear dynamic inversion and the backstepping techniques is briefly introduced. Then the flight path dynamics for a transportation aircraft are displayed. Following the detailed flight-path tracking control law synthesis from both techniques are displayed. Then respective simulation results are provided and their performances are compared.

\section{Nonlinear Dynamic Inversion}

The basic idea of nonlinear dynamic inversion [3] [4] [5] is to transform a nonlinear dynamic system into an equivalent controllable linear system through a change of coordinates by compensating the nonlinear parts of system dynamics. This leads to adopt as control objective a stable linear dynamics for the output error. A nonlinear state feedback control law is then derived when a non singularity condition is satisfied.

Consider an affine dynamic system given by:

$$
\begin{gathered}
\dot{x}=f(x)+\sum_{i=1}^{m} g_{i}(x) u_{i} \\
y=h(x)
\end{gathered}
$$

where $x \in \mathbf{R}^{n}, u \in \mathbf{R}^{m}, y \in \mathbf{R}^{m}$, and $f, g$ and $h$ are smooth vector fields of $x$. The system has relative degrees $r_{i}\left(\sum_{i=1}^{m} r_{i} \leq n, i=1, \ldots, m\right)$ around the point $x_{0}$ corresponding to the outputs $y_{i}$ at $x_{0}$ such that

$$
\left(\begin{array}{c}
y_{1}^{\left(r_{1}\right)} \\
\vdots \\
y_{m}^{\left(r_{m}\right)}
\end{array}\right)=A(x)+B(x) u
$$

If $B(x)_{m \times m}$ is nonsingular, the resulted feedback control law can be therefore obtained:

$$
u=B^{-1}(x)(v-A(x))
$$

where new control input $v=\left[v_{1}, \ldots, v_{m}\right]^{\mathrm{T}}$ can be chosen by

$$
v_{i}=y_{d}^{\left(r_{i}\right)}-\sum_{k=0}^{r_{i}-1} c_{i k}\left(y_{i}^{(k)}-y_{d i}^{(k)}\right)
$$

where $y_{d i}$ is the reference control input for the output dynamics. The tracking error is given by

$$
e_{i}=y_{i}-y_{d i}
$$

whose dynamics is given by :

$$
e_{i}^{\left(r_{i}\right)}+c_{i r_{i}-1} e_{i}^{\left(r_{i}-1\right)}+\cdots+c_{i 1} e_{i}^{(1)}+c_{i 0} e_{i}=0
$$

where the coefficients $c_{i k}$ can be chosen to make the output dynamics asymptotically stable and ensure the tracking of output $y_{i}$ towards the reference output $y_{d i}$. However the derived feedback control law works only if no internal dynamics $\left(\sum_{i=1}^{m} r_{i}=n\right)$ are present or its internal dynamics are stable $\left(\sum_{i=1}^{m} r_{i}<n\right)$.

\section{Backstepping}

Consider a dynamic system

$$
\begin{gathered}
\dot{x}_{1}=f\left(x_{1}\right)+g\left(x_{1}\right) x_{2} \\
\dot{x}_{2}=u
\end{gathered}
$$

where $x_{1} \in \mathrm{R}, \mathrm{x}_{2} \in \mathrm{R}$ are state variables and $\mathrm{u} \in \mathrm{R}$ is the control input.

The control objective [6] [7] [8] to this dynamic system is to design a control law such that the state $\mathrm{x} 1$ can be stabilized at 0 , a supposed global asymptotically stable equilibrium with null input 
$(f(0)=0)$. Furthermore, it can observed that $\mathrm{x}_{2}$ can be regarded as a virtual (or mediate) control input for the dynamics $\mathrm{x}_{1}$, and the dynamics of $\mathrm{x}_{2}$ is controlled by the real control input $u$. This is an important feature to make use of for the following control law synthesis. Now, suppose that there exists a control law:

$$
x_{2}=\xi\left(x_{1}\right)
$$

such that the dynamics of $x_{1}$ can be stabilized at 0 while we can find a Lyapunov function $V_{1}\left(x_{1}\right)$ satisfy the condition

$$
\dot{V}_{1}\left(x_{1}\right)=\frac{\partial V_{1}}{\partial x_{1}}\left(f\left(x_{1}\right)+g\left(x_{1}\right) \xi\left(x_{1}\right)\right) \leq-W_{1}\left(x_{1}\right)
$$

where $W_{1}\left(x_{1}\right)$ is positive definite function. Then the whole dynamics can be expressed as:

$$
\begin{gathered}
\dot{x}_{1}=f\left(x_{1}\right)+g\left(x_{1}\right) \xi\left(x_{1}\right)+g\left(x_{1}\right) z \\
\dot{z}=w
\end{gathered}
$$

where

$$
\begin{gathered}
z=x_{2}-\xi\left(x_{1}\right) \\
w=u-\dot{\xi}\left(x_{1}\right)
\end{gathered}
$$

with

$$
\dot{\xi}\left(x_{1}\right)=\frac{\partial \xi}{\partial x_{1}}\left(f\left(x_{1}\right)+g\left(x_{1}\right) x_{2}\right)
$$

Then, the Lyapunov function candidate of the full system is given by:

$$
V\left(x_{1}, z\right)=V_{1}\left(x_{1}\right)+\frac{1}{2} z^{2}
$$

The time derivative of $V(x, z)$ is given by:

$$
\dot{V}\left(x_{1}, z\right)=\frac{\partial V_{1}}{\partial x_{1}}\left(f\left(x_{1}\right)+g\left(x_{1}\right) \xi\right)+\frac{\partial V_{1}}{\partial x_{1}} g\left(x_{1}\right) z+z w
$$

Substituting the inequality of Eq.(9) and Eq.(10) into Eq.(14) yields:

$$
\dot{V}\left(x_{1}, z\right) \leq-W_{1}\left(x_{1}\right)+\frac{\partial V_{1}}{\partial x_{1}} g\left(x_{1}\right) z+z w
$$

By an adequate choice of $w$, such as:

$$
w=-\frac{\partial V_{1}}{\partial x_{1}} g\left(x_{1}\right)-k z
$$

where $k$ is a positive constant, the full system is globally asymptotically stable since it satisfies the following condition:

$$
\dot{V}\left(x_{1}, z\right) \leq-W_{1}\left(x_{1}\right)-k z^{2}
$$

Finally, substituting Eq.(7) and Eq.(11) into Eq.(16) yields the effective control input:

$u=\frac{\partial \xi\left(x_{1}\right)}{\partial x_{1}}\left(f\left(x_{1}\right)+g\left(x_{1}\right) x_{2}\right)-\frac{\partial V_{1}}{\partial x_{1}} g\left(x_{1}\right)-k\left(x_{2}-\xi\left(x_{1}\right)\right)$

\section{Dynamics Equations}

In general, the flight path angle of an aircraft is defined as:

$$
\gamma=\sin ^{-1}\left(\frac{\dot{h}}{V_{T}}\right)
$$

where $\dot{h}$ and $V_{\mathrm{T}}$ respectively represent the change rate of altitude change and the true airspeed. Restricting the aircraft dynamics to the vertical plane, additional assumptions of non-rolling motion (roll angle: $\psi=0$ ) and negligible sideslip (sideslip angle: $\beta=0$ ) are done. Then:

$$
\begin{gathered}
\gamma=\theta-\alpha \\
\dot{\theta}=q
\end{gathered}
$$

$$
q_{W}=q
$$

where $\theta, \alpha, q$ and $q_{W}$ is respectively the pitch angle, the angle-of-attack, the pitch rate in body-axes, and the pitch rate in wind-axis. The transport aircraft in general operates at low angle-of-attack such that:

$$
\sin \alpha \approx \alpha
$$


The time-derivative of the flight path angle is therefore approximated by:

$$
\dot{\gamma}=-\frac{g}{V_{T}} \cos \gamma+\frac{L}{m V_{T}}
$$

where $V_{\mathrm{T}}$ is the airspeed of aircraft; $L$ is the lift; $m$ and $g$ are the mass and the gravitational acceleration respectively. The relevant dynamic equations of $\alpha$ and $\theta$ are given by:

$$
\begin{gathered}
\dot{\alpha}=-\frac{1}{m V_{T}}(L-m g \cos \gamma)+q \\
\dot{q}=\frac{M}{I_{y}}
\end{gathered}
$$

where $M$ is the pitching moment and $I_{y}$ is the inertial moment about $y$ axis in body-axes. The aerodynamic force and moment can be expressed as:

$$
\begin{gathered}
L=\frac{\rho V_{T}^{2} S}{2}\left(C_{L_{0}}+C_{L_{\alpha}} \alpha+C_{L_{\delta}} \delta_{e}\right) \\
M=\frac{\rho V_{T}^{2} S c}{2}\left[C_{m_{0}}+C_{m_{\alpha}} \alpha+C_{m_{\delta}} \delta_{e}\right. \\
\left.+\frac{c}{2 V_{T}}\left(C_{m_{q}} q+C_{m_{\dot{\alpha}}} \dot{\alpha}\right)\right]
\end{gathered}
$$

where $\rho$ is the air density; $\bar{c}$ is the mean wing chord length; $\delta_{e}$ is the deflection angle of elevator; $C_{L^{(*)}}$ and $C_{m\left({ }^{*}\right)}$ are the lift coefficients and the pitching moment coefficients respectively from different contributions.

\section{Control Law Synthesis}

\section{Nonlinear dynamic Inversion}

In this study, the system to be controlled is considered as a SISO system: the flight-path angle $(\gamma)$ is the output while the input is the deflection of the elevator $\left(\delta_{\mathrm{e}}\right)$. The airspeed $\mathrm{V}_{\mathrm{T}}$ and the thrust $\mathrm{T}$ are assumed to remain constant while flaps adopt a fixed position. From Eq.(22) - Eq.(23), the state and output of aircraft dynamics can be chosen such as:

$$
S:\left\{\begin{array}{l}
x=(\gamma, \alpha, q)^{T} \\
u=\delta_{e} \\
y=\gamma
\end{array}\right.
$$

where $x, u, y$ respectively denote the entire state, the control input and the output of the system.

By differentiating the output until the input appears, it is obvious that the control input $\left(\delta_{\mathrm{e}}\right)$ appears in the first time-derivative of the output $(\gamma)$ such that its relative degree equals to 1 so that second order unobservable internal dynamics is present. This internal dynamics includes the fast dynamics of pitching rate. At this stage, the direct implementation of dynamic inversion technique may not work due to this internal dynamics. In order to overcome this internal dynamics, the input can be changed to elevator rate reference value so that the differentiating process can be pursued. Differentiating again the output yields:

$$
\ddot{\gamma}=\frac{g}{V_{T}} \dot{\gamma} \sin \gamma+\frac{\rho V_{T} S}{2 m}\left(C_{L_{\alpha}} \dot{\alpha}+C_{L_{\delta}} \dot{\delta}_{e}\right)
$$

For further simplicity, assume:

$\sin \gamma=\gamma$ and $\dot{\alpha}=q-\dot{\gamma}$

such that:

$$
\ddot{\gamma}=\frac{g}{V_{T}} \dot{\gamma}+\frac{\rho V_{T} S}{2 m}\left[C_{L_{\alpha}}(q-\dot{\gamma})+C_{L_{\delta}} \dot{\delta}_{e}\right]
$$

Since at this stage internal dynamics are not eliminated, another differentiation appears necessary. Considering that the elevator dynamics obeys to the first order relation:

$$
\ddot{\delta}_{e}=\frac{1}{\tau_{E}}\left(\dot{\delta}_{e c}-\dot{\delta}_{e}\right)
$$

where $\dot{\delta}_{e c}$ is the control signal, it is feasible to consider an additional differentiation. This gives:

$$
\gamma^{(3)}=\frac{g}{V_{T}}\left(\ddot{\gamma}+\dot{\gamma}^{2}\right)+\frac{\rho V_{T} S}{2 m}\left[C_{L_{\alpha}}(\dot{q}-\ddot{\gamma})+C_{L_{\delta}} \ddot{\delta}\right]
$$


Then the nonsingular condition reduces here to:

$\frac{\rho V_{T} S}{2 m} C_{L_{\delta}} \neq 0$

which is satisfied, although $C_{L_{\delta}}$ may be very small.

Here it is supposed that the path-angle control objective is to follow a given trajectory $\gamma_{d}$ in such a way that output error is stable and obeys the third order linear dynamics:

$$
\begin{aligned}
& \left(\gamma^{(3)}-\gamma_{d}^{(3)}\right)+c_{2}\left(\ddot{\gamma}-\ddot{\gamma}_{d}\right)+c_{1}\left(\dot{\gamma}-\dot{\gamma}_{d}\right)+c_{0}\left(\gamma-\gamma_{d}\right)=0 \\
& \dot{\delta}_{e c}=\dot{\delta}_{e}+\frac{\tau_{e} m V_{T}}{C_{L_{\delta}}}\left\{\frac{g}{V_{T}}(\dot{\gamma})^{2}+\left(\frac{g}{V_{T}} \gamma-\frac{1}{m V_{T}}\left(\frac{1}{2} \rho V_{T}^{2} S C_{\mathrm{L}_{\alpha}}\right)\right) \ddot{\gamma}\right. \\
& +\frac{1}{m V_{T}}\left(\left(\frac{1}{2} \rho V_{T}^{2} S C_{\alpha_{\alpha}}\right) \frac{M}{I_{y}}\right) \\
& \left.+\gamma_{d}^{(3)}-c_{2}\left(\ddot{\gamma}-\ddot{\gamma}_{d}\right)-c_{1}\left(\dot{\gamma}-\dot{\gamma}_{d}\right)-c_{0}\left(\gamma-\gamma_{d}\right)\right\}
\end{aligned}
$$

Observe that, since the actuator dynamics have been introduced, internal dynamics, related with the angle of attack, remains with a risk of instability. Observe also that to implement this control law it is be necessary to get accurate estimates of the current pitch moment.

Another possible approach to implement the nonlinear inversion technique is to adopt simplified aerodynamic models and introduce a corrective term. Then, considering only the main aerodynamic effects, relations (24a) and (24b) become:

$$
\begin{gathered}
L \approx \frac{\rho V_{T}^{2} S}{2}\left(C_{L_{0}}+C_{L_{\alpha}} \alpha\right) \\
M \approx \frac{\rho V_{T}^{2} S \bar{c}}{2}\left[C_{m_{0}}+C_{m_{\alpha}} \alpha\right]
\end{gathered}
$$

Relation (28) becomes:

$$
\ddot{\gamma}=\frac{g}{V_{T}} \dot{\gamma}+\frac{\rho V_{T} S}{2 m}\left[C_{L_{\alpha}}(q-\dot{\gamma})\right]
$$

and relation (30) becomes:

$$
\gamma^{(3)}=\frac{g}{V_{T}}\left(\ddot{\gamma}+\dot{\gamma}^{2}\right)+\frac{\rho V_{T} S}{2 m}\left[C_{L_{\alpha}}(\dot{q}-\dot{\gamma})\right]
$$

The new control law is given by:

$$
\begin{aligned}
& \delta_{e}=\frac{C_{m_{0}}+C_{m_{\alpha}} \alpha}{C_{m_{\delta}}}+\left(\frac{4 m I_{y}}{C_{m_{\delta}}\left(\rho V_{T} S\right)^{2}}\right)\left\{-\frac{g}{V_{T}}(\ddot{\gamma} \gamma+\dot{\gamma})^{2}+\right. \\
& \left.+\gamma_{d}^{(3)}-c_{2}\left(\ddot{\gamma}-\ddot{\gamma}_{d}\right)-c_{1}\left(\dot{\gamma}-\dot{\gamma}_{d}\right)-c_{0}\left(\gamma-\gamma_{d}\right)\right\}
\end{aligned}
$$

However, since in this case important simplifications have been adopted to avoid the appearance of internal dynamics, it appears convenient to introduce an integral corrective term to get a control law such as:

$$
\delta_{e c}=\delta_{e c}-c_{3}\left(\frac{4 m I}{C_{m_{\delta}}\left(\rho V_{T} S\right)^{2}}\right)_{0}^{t}\left(\gamma-\gamma_{d}\right) d \tau
$$

So it appears that, whatever the approach chosen, the nonlinear inversion technique leads to foreseeable difficulties. They will be displayed through simulations in the case of the path angle control of an F16.

\section{Application of backstepping to flight-path angle control}

As considered earlier, the lift contributed from the deflection of elevator is relatively small in comparison with the contribution from the angle-ofattack $\left(C_{L \delta} \leq 10 \% C_{L \alpha}\right)$. When the term of the deflection of elevator is neglected, the dynamics of flight-path angle are written as:

$$
\begin{aligned}
\dot{\gamma} & \approx-\frac{g}{V_{T}} \cos \gamma+\frac{L}{m V_{T}} \\
& =\Gamma\left(m, V_{T}, \alpha, \gamma\right)
\end{aligned}
$$

The first step of a backstepping approach consists in defining the output error which is given here by:

$$
z_{\gamma}=\gamma-\gamma_{d}
$$

where $\gamma_{d}$ is the desired flight-path angle. Then the error dynamics of flight-path angle is given by:

$$
\dot{z}_{\gamma}=\Gamma(m, V, \alpha, \gamma)
$$


A Lyapunov function for $\gamma$ is given by:

$$
V_{\gamma}=\frac{1}{2} z_{\gamma}^{2}
$$

Its time-derivative is then given by

$$
\begin{aligned}
\dot{V}_{\gamma} & =z_{\gamma} \Gamma(m, V, T, \alpha, \gamma) \\
& =z_{\gamma} \Gamma\left(m, V, T, \theta-\gamma_{c}-z_{\gamma}, \gamma_{c}+z_{\gamma}\right)
\end{aligned}
$$

where it is possible to choose a proper $\theta_{c}$ for $\theta$ such as:

$$
\theta_{c}=\gamma+\lambda_{\gamma} z_{\gamma}+\mu_{\gamma} \int z_{\gamma} d \tau \text { with } \lambda_{\gamma}<1
$$

which makes the dynamics of $\gamma$ asymptotically stable since $z_{\gamma}$ obeys approximately to equation:

$$
\ddot{z}_{\gamma}-\left(\frac{\rho V_{T}^{2} S C_{L_{\alpha}}}{2}\right)\left(\lambda_{\gamma} \dot{z}_{\gamma}+\mu_{\gamma} z_{\gamma}\right) \approx 0
$$

such that:

$$
\dot{V}_{\gamma}=\Gamma\left(m, V, T,\left(\lambda_{\gamma}-1\right) z_{\gamma}+\mu_{\gamma} \int z_{\gamma} d \tau, \gamma_{c}+z_{\gamma}\right)<0
$$

At the second step, the dynamics of pitch angle $\theta$ evidently are related to the pitching rate $q$ by Eq.(20b). The pitch dynamics here is assumed to be first order:

$$
q_{c}=\frac{\theta-\theta_{c}}{\tau_{\theta}}
$$

where $\tau_{\theta}$ is a time constant.

At the third step, a connection is built between the pitching rate and the deflection of elevator $\delta_{e}$ by using the dynamic equation of pitching moment which is here the source of most modeling errors.

By defining the error dynamics of $q$ such as:

$$
z_{q}=q-q_{c}
$$

A Lyapunov function for $q$ can be chosen such as

$$
V_{q}=V_{\gamma}+\frac{1}{2} z_{q}^{2}
$$

Using Eq.(39), Eq.(41) and Eq.(42) in Eq.(43) gives:

$$
V_{q}=V_{\gamma}+\frac{1}{2}\left(q-\frac{\gamma_{c}-\left(\lambda_{\gamma} z_{\gamma}+\mu_{\gamma} \int z_{\gamma} d \tau\right.}{\tau_{\theta}}\right)^{2}
$$

Its time derivative is given by:

$$
\dot{V}_{q}=\dot{V}_{\gamma}+\left(q-\frac{\gamma_{c}-\lambda_{\gamma} z_{\gamma}-\mu_{\gamma} \int z_{\gamma} d \tau}{\tau_{\theta}}\right)\left(\frac{M}{I_{y}}+\frac{\lambda_{\gamma}}{\tau_{\theta}} \Gamma\left(m, V_{T}, \alpha, \gamma\right)\right)
$$

where a proper $\delta_{e}$ can be chosen such as:

$$
\frac{M}{I_{y}}=\frac{\lambda_{\gamma}}{\tau_{\theta}} \Gamma(m, V, T, \alpha, \gamma)-\mu\left(q-\frac{\gamma_{c}-\lambda_{\gamma} z_{\gamma}-\mu_{\gamma} \int z_{\gamma} d \tau}{\tau_{\theta}}\right)
$$

where $\mu$ is a positive parameter.

The pitching dynamics are then stabilized since they satisfy the following condition:

$$
\dot{V}_{q}<0
$$

since:

$$
\dot{V}_{q}=\dot{V}_{T \gamma}-\mu\left(q-\frac{\gamma_{c}-\lambda_{\gamma} z_{\gamma}-\mu_{\gamma} \int z_{\gamma} d \tau}{\tau_{\theta}}\right)^{2}
$$

By substituting Eq.(23) and Eq.(24) into Eq.(38) we can finally derive the Backstepping control law:

$$
\begin{aligned}
& \left.\delta_{e}=\frac{-2 I_{y}}{\rho V_{T}^{2} S c C_{m_{\delta}}}\left[\lambda \Gamma\left(m, V_{T}, \alpha, \gamma\right)+\mu q-\frac{\gamma_{c}-\lambda_{\gamma} z_{\gamma}-\mu_{\gamma} \int z_{\gamma} d \tau}{\tau_{\theta}}\right)\right] \\
& -\frac{1}{C_{m_{\delta}}}\left[C_{m_{0}}+C_{m_{\alpha}} \alpha+\frac{c}{2 V_{T}}\left(C_{m_{q}} q+C_{m_{\dot{\alpha}}} \dot{\alpha}\right)\right]
\end{aligned}
$$

It appears that the backstepping approach is far from being straightforward and that such an intricate construction should be tested through fully to provide confidence in its performances.

\section{Simulation Results}

A general 6-DOF nonlinear dynamics of the F16 combat aircraft including the first-order 
dynamics of actuators and propulsion model is used for simulation []. In order to compare the robustness of both path angle control techniques., a rough PID airspeed controller is implemented to maintain speed around a reference value. The F-16 is assumed to fly at a speed of $500 \mathrm{ft} / \mathrm{s}$ with initial altitude of $20000 \mathrm{ft}$.

It appears necessary to tune the controller gains with a trial-and-error process to obtain an acceptable tracking performance.

Figure 1 and figure 2 show the tracking performance of flight-path angle and relevant data when using the proposed modified nonlinear dynamic inversion technique while the airspeed controller is engaged. However, the airspeed changes during the engagement of the control input. This results in a degradation of the tracking performance. The case shown in figure 3 and figure 4 is performed without the airspeed controller engaged such as to severely affect the tracking performance while the airspeed changes autonomously with the fixed thrust.

Figures 5-8 display the tracking performance obtained using the backstepping control technique. The response appears to be smoother than the one obtained with the nonlinear dynamic inversion technique.
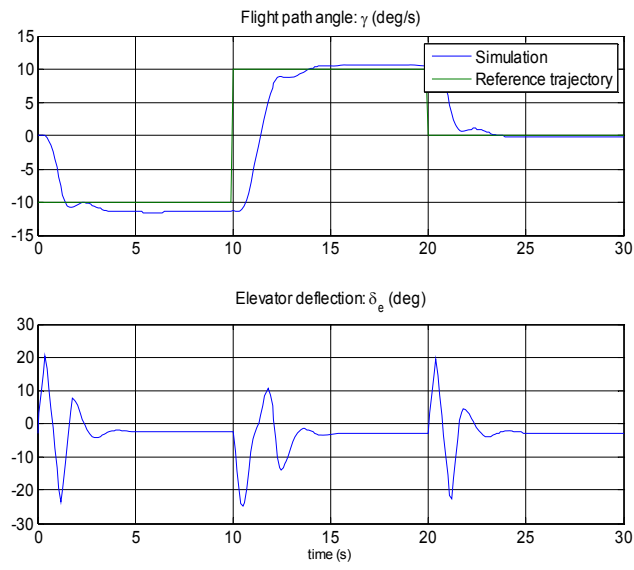

Figure 1. NLI path angle control with airspeed regulation
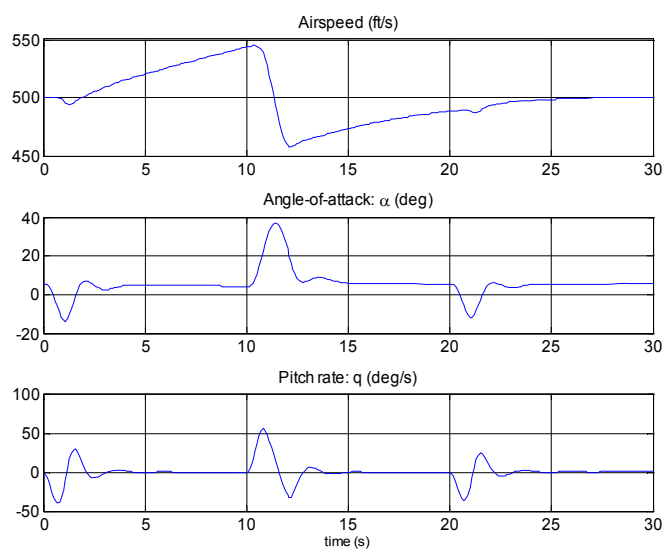

Figure 1. NLI path angle control with airspeed regulation
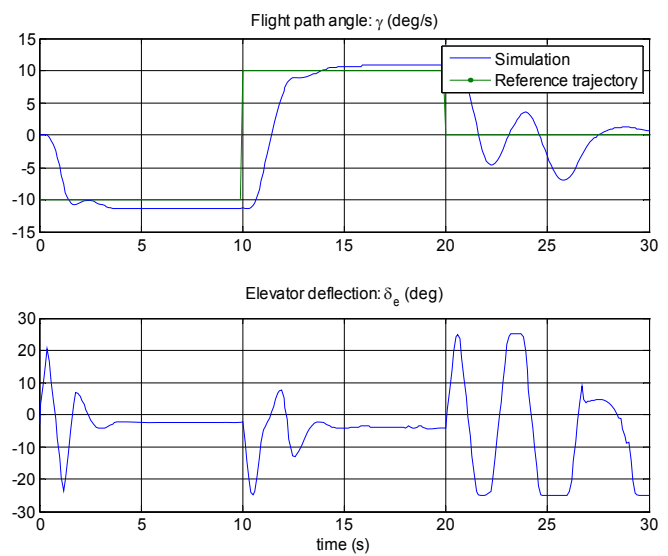

Figure 3. NLI path angle control without airspeed regulation
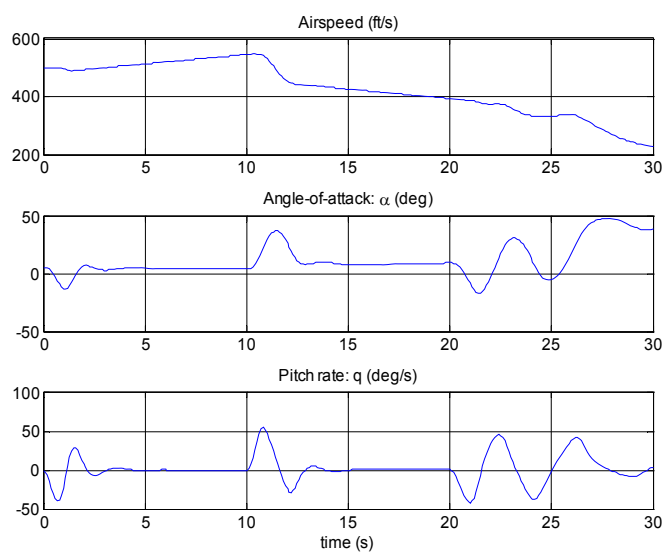

Figure 4. NLI path angle control without airspeed regulation 

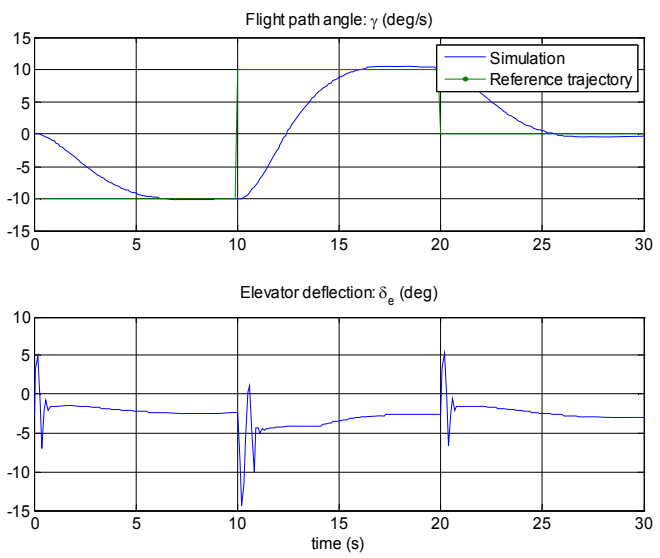

Figure 5. Backstepping path angle control without airspeed regulation
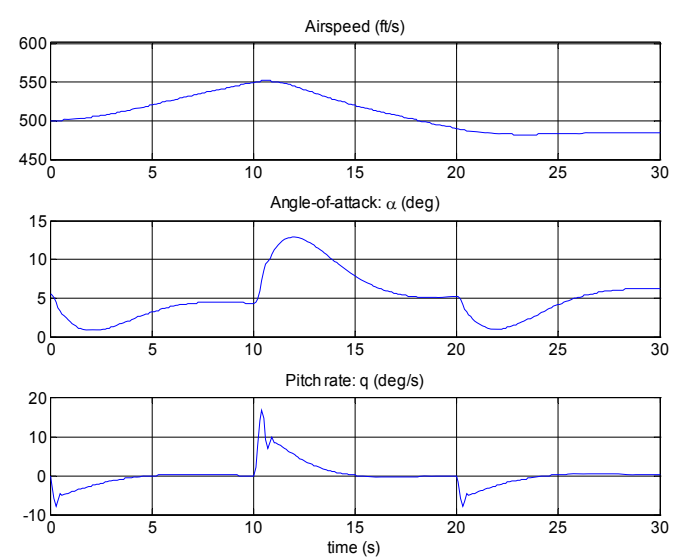

Figure 6. Backstepping path angle control without airspeed regulation
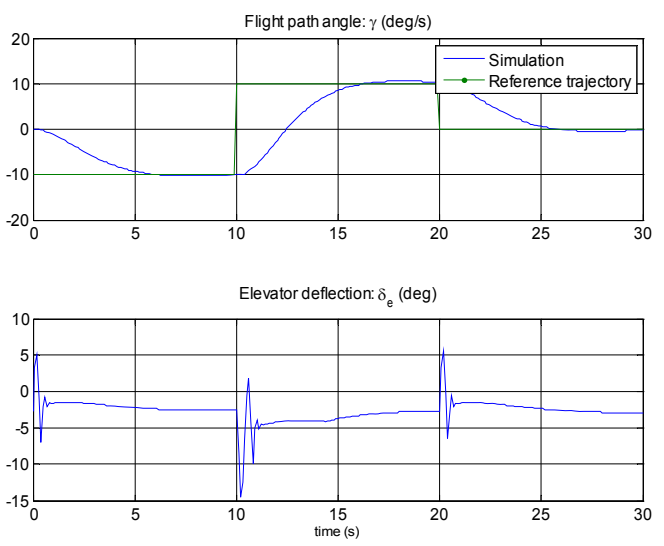

Figure 7. Backstepping path angle control with airspeed regulation
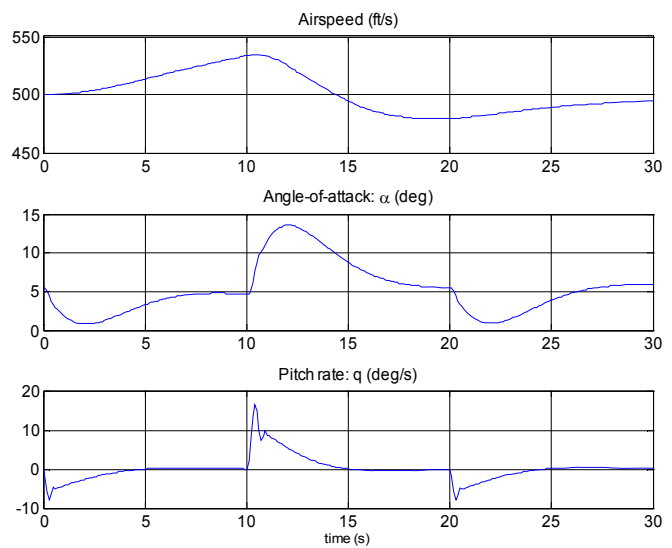

Figure 8. Backstepping path angle control with airspeed regulation

It appears that the results obtained with the backstepping technique are more robust to airspeed variations. This technique presents also more tuning parameters related with the successive Lyapunov functions used to secure the global stability of the controlled system.

\section{Conclusion}

In this paper the problem of path angle control design for an aircraft has been considered. Two popular non linear control techniques have been introduced and applied to this control problem: nonlinear dynamics inverse control and backstepping.

In the case of non linear dynamics inverse control, it appears that the choice of the longitudinal dynamics model which is used in the inversion, is critical to avoid the presence of uncontrolled internal dynamics. This choice may lead, when important simplifying assumptions are made, to the introduction of an integral term to improve the accuracy of the path angle tracking.

Also, it appears that the application of the backstepping technique is not straightforward and that the resulting complex control structure must be intensively tested to provide confidence in its performances. Indeed, the stability of the control scheme is here no more guaranteed in theoretical grounds considering the assumptions which have been necessary to be done to apply this multilevel control scheme. 


\section{References}

[1] Khalil, H., "Non Linear Systems," $2^{\text {nd }}$ Edition, Prentice Hall, Upper Saddle River, NJ, 2002.

[2] Sira-Ramirez, H., R. Marquez, F. RivasEcheverria and O. Lanes-Santiago, "Control de Sistemas no Lineares," Pearson Educacion S.A., Madrid, 2005.

[3] Lane, S.H., R.F. Stengel, "Flight Control Design Using Non-Linear Inverse Dynamics," Automatica, Vol.24, pp.471-483, 1988.

[4] Enns, D., D. Bugajski, R. Hendrick, G. Stein, "Dynamic Inversion: an Evolving Methodology for Flight Control Design," International Journal of Control, Vol. 1, pp. 71-91, 1994.

[5] Kolesnikov, E., "NDI-Based Flight Control Law Design," AIAA paper 5977, 2005.

[6] Lu, W.C., "Contribution au Suivi Automatique de Trajectoires par un Avion, Commande Plate et Réseaux de Neurones," PhD Thesis, LAAS du CNRS, Toulouse, 2005.
[7] Glad, T., O. Harkerdard, "Backstepping Control of a Rigid Body," Proc. CDC, Las Vegas, N.V., 2002.

[8] Robinson, J.W.C., U. Nilson, "Design of a Nonlinear Autopilot for Velocity and Attitude Control using Block Backstepping," AIAA paper 6266, 2005.

\section{Email}

lili.duan-ext@enac.fr

wclu@,laas.fr

thierry.miquel@cena.fr

felix.mora@enac.fr

25th Digital Avionics Systems Conference October 15, 2006 